\title{
Application of MALDI-TOF MS for requalification of a Candida clinical isolates culture collection
}

\author{
Reginaldo Lima-Neto ${ }^{1,2}$, Cledir Santos ${ }^{2}$, Nelson Lima ${ }^{2}$, Paula Sampaio ${ }^{3}$, \\ Célia Pais ${ }^{3}$, Rejane P. Neves ${ }^{1}$ \\ ${ }^{1}$ Department of Mycology, Centre of Biological Sciences, Federal University of Pernambuco, \\ Recife, PE, Brazil. \\ ${ }^{2}$ Micoteca da Universidade do Minho, Centre of Biological Engineering, \\ University of Minho, Braga, Portugal. \\ ${ }^{3}$ Department of Biology, Centre of Molecular and Environmental Biology, \\ University of Minho, Braga, Portugal.
}

Submitted: December 18, 2012; Approved: September 9, 2013.

\begin{abstract}
Microbial culture collections underpin biotechnology applications and are important resources for clinical microbiology by supplying reference strains and/or performing microbial identifications as a service. Proteomic profiles by MALDI-TOF MS have been used for Candida spp. identification in clinical laboratories and demonstrated to be a fast and reliable technique for the routine identification of pathogenic yeasts. The main aim of this study was to apply MALDI-TOF MS combined with classical phenotypic and molecular approaches to identify Candida clinical isolates preserved from 1 up to 52 years in a Brazilian culture collection and assess its value for the identification of yeasts preserved in this type of collections. Forty Candida spp. clinical isolates were identified by morphologi$\mathrm{cal}$ and biochemical analyses. Identifications were also performed by the new proteomic approach based on MALDI-TOF MS. Results demonstrated 15\% discordance when compared with morphological and biochemical analyses. Discordant isolates were analysed by ITS sequencing, which confirmed the MALDI-TOF MS identifications and these strains were renamed in the culture collection catalogue. In conclusion, proteomic profiles by MALDI-TOF MS represents a rapid and reliable method for identifying clinical Candida species preserved in culture collections and may present clear benefits when compared with the performance of existing daily routine methods applied at health centres and hospitals.
\end{abstract}

Key words: BRCs, Candida, clinical yeasts, culture collections, MALDI-TOF MS.

\section{Introduction}

Culture collections play a key role in microbiology, since they are responsible for gathering and preserving well characterised strains. In addition, culture collections are responsible for ex situ preservation of microbial resources and their related information. In fact, each single microbial strain has not great value unless it is very well characterised with the current state of the art applied for each microbial group and the related information available in the appropriate format (Boundy-Mills, 2012).
The scientific community and the end users demand from the culture collections authentic biological materials with reproducible properties that allow them to use the strains to fit their needs: as type strains for taxonomic proposes, reference strains as standards, or even unique strains for research and exploitation of their peculiar properties (Smith, 2012; Simões et al., 2013). The current challenges created by the OECD best practices guidelines for Biological Resource Centres (BRCs) generated an unprecedented wave in culture collections to adopt new practices and methods in order to guarantee high quality operational stan-

Send correspondence to C. Santos. Micoteca da Universidade do Minho, Institute for Biotechnology and Bioengineering, Centre of Biological Engineering, University of Minho, Campus de Gualtar, 4710-057 Braga, Portugal. E-mail: cledir.santos@deb.uminho.pt. 
dards and provide authentic materials (OECD, 2007; Schoch et al., 2012).

The historical Brazilian fungal service culture collection University Recife Mycology (URM, www.ufpe.br/micoteca) was established by Prof. Chaves Batista in 1954 and is hosted by the Department of Mycology of the Federal University of Pernambuco (Recife, Brazil). Since the number and clinical impact of severe infections due to yeast species has increased the identification of yeast species with clinical relevance is one among several significant services offered by the URM culture collection. In addition, the sub-set collection of Candida species is of relevance due to possibility of supplying reference strains for clinical studies.

Candida species emerged as the major opportunistic pathogens in immunocompromised patients, and currently constitute one of the most common causes of nosocomial infections in intensive care units (Marklein et al., 2009). Currently, this genus comprises more than 160 species, is expanding rapidly and is under a continuous taxonomic revision. Approximately 20 species cause disease in humans, although the number of new reports is increasing (Qian et al., 2008; Putignani et al., 2011). Candidemia is the fourth most common cause of hospital bloodstream infection worldwide being associated with extended hospital stays and high mortality rates among critically ill patients (Medrano et al., 2006). The case/fatality rate is approximately $34 \%$ with an estimated 72.8 million worldwide opportunistic Candida infections per year (Marklein et al., 2009).

The time required for pathogen identification is an important determinant in the infection-related mortality rates in hospitalised patients. Death rates and costs associated with infectious diseases could significantly be reduced by employing rapid identification techniques (Essendoubi et al., 2005). Inevitably, non-Candida yeasts that can act as human pathogens have been misidentified as Candida species. Their identification may pose a challenge when the type strains have not yet been included into the diagnostic databases of any semi- or full-automated identification system (Lockhart et al., 2008; Marklein et al., 2009; Putignani et al., 2011). Several reports have addressed the difficulty to identify yeast strains to the species level by conventional methods, since they are highly dependent on variables such as growth medium and temperature (Latouche et al., 1997; Mozina and Raspor, 1997; Correia et al., 2006; Leaw et al., 2006; Qian et al., 2008) and, in some limit cases, the interpretation of the results remain very subjective. This makes both molecular biology and spectral approaches crucial when the mycologist faces an isolate with unusual traits.

Matrix Assisted Laser Desorption/Ionisation TimeOf-Flight Mass Spectrometry (MALDI-TOF MS) is a physic-chemical technique that has been used for rapid and reliable microbial identification. The spectrum generated is analysed as an individual proteomic profile with the molecular mass ranging from 2000 to $20000 \mathrm{Da}$, where important ribosomal proteins appear, which is an advantage because they can be readily employed as biomarkers (Santos et al., 2010a, 2011; Passarini et al., 2013). Proteomic profiles by MALDI-TOF MS for rapid identification of intact bacteria were firstly used in 1996 (Holland et al., 1996). More recently MALDI-TOF MS has been used to detect and identify yeasts. It is able to discriminate closely related species, such as $C$. glabrata from C. bracarensis, C. albicans from C. dubliniensis and, C. metapsilosis and C. orthopsilosis from C. parapsilosis, previously separated only by molecular methods (Sullivan et al., 1995; Tavanti et al., 2005; Correia et al., 2006; van Veen et al., 2010; Jensen and Arendrup, 2011; Marinach-Patrice et al., 2010; Putignani et al., 2011; Santos et al., 2011) or Fourier Transform Infrared (FT-IR) techniques (Santos et al., 2010b).

The purpose of the present study was to apply MALDI-TOF MS combined with classical phenotypic and molecular approaches to identify Candida clinical isolates preserved from 1 up to 52 years in a Brazilian culture collection and assess its value for the identification of yeasts preserved in this type of service collections.

\section{Materials and Methods}

\section{Strains and growth conditions}

All 40 Candida isolates analysed in this study were obtained from the URM culture collection (www.ufpe.br/micoteca). This set of yeasts comprises all clinical isolates of this genus available at URM. Escherichia coli strain $\mathrm{DH} 5 \alpha$ was used as standard for the MALDI-TOF MS external calibration and was obtained from the Micoteca da Universidade do Minho (MUM, www.micoteca.deb.uminho.pt). Trichophyton rubrum MUM 09.09 was incorporated into the analysis as an outgroup. All cultures were preserved at $-80^{\circ} \mathrm{C}$ and cryovials were thawed, opened and the strains sub-cultured according to the instructions issued by URM and MUM culture collections. Homogenous inocula of yeast cells were grown and maintained on Yeast Extract Peptone Dextrose agar medium (YEPD). Escherichia coli cells were grown and maintained on Luria-Bertani agar medium (LB). Incubations were standardised at $20 \mathrm{~h}$ and strains were grown aerobically at $37^{\circ} \mathrm{C}$. In order to avoid changes in the protein expression pattern the culture conditions and growth time were standardised as described above. All cultures were checked for purity prior to use and were sub-cultured at least twice prior to MALDI-TOF MS analysis.

\section{Classical phenotypic identification}

The isolates were identified by macro- and micromorphology. The colour, shape, topology of each colony and the cell morphology were considered using Sabouraud dextrose agar medium with $2.5 \%$ yeast extract (Difco, USA). The induction of sexual reproduction structure using Gorodkowa agar (Difco, USA) was performed. Chlamy- 
dospores were induced on bile agar (Difco, USA) and scored as present or absent after 3 days at $25^{\circ} \mathrm{C}$. The isolates were tested biochemically by carbohydrate assimilation and fermentation assays. The production of urease and acetic acid were also assessed using urea and calcium carbonate media (Difco, USA), respectively. The maximum temperature of growth for each isolate was determined according Barnett et al. (2000) and De Hoog et al. (2000). The isolates which presented ambiguous identification were further analysed by VITEK-2 XL (bioMerieux, Brazil) according to previous reports (Macedo et al., 2009).

\section{MALDI-TOF MS plate preparation}

Using a yellow tip a tiny sample (about $10^{6}$ yeasts per sample) was transferred directly from a single colony and spotted onto the 48 well flex target plate (FlexiMass, Shimadzu Biotech, UK). Aliquots of $0.5 \mu \mathrm{L}$ of $25 \%$ formic acid were added and mixed gently with the yeasts. When the liquid medium was almost evaporated $0.5 \mu \mathrm{L}$ matrix solution $(75 \mathrm{mg} / \mathrm{mL}$ 2,5-dihydroxybenzoic acid [DHB] in ethanol/water/acetonitrile $[1: 1: 1 ; \mathrm{v} / \mathrm{v} / \mathrm{v}]$ with $0.03 \%$ trifluoroacetic acid [TFA]) was added and mixed gently. The matrix solution addition was performed without the additional treatment with formic acid for E. coli. All solutions were freshly prepared and stored at $5{ }^{\circ} \mathrm{C}$ during the work. In order to assess the quality and reproducibility of the spectral data, each yeast isolate was analysed in duplicate. All samples were air dried at room temperature and finally analysed by MALDI-TOF MS.

\section{MALDI-TOF MS data acquisition}

The analyses were performed on an Axima LNR system (Kratos Analytical, Shimadzu, UK) equipped with a nitrogen laser $(337 \mathrm{~nm})$, where the laser intensity was set just above the threshold for ion production. Twelve defined ribosomal proteins of intact E. coli DH5 $\alpha$ cells (4365.4, 5096.8, 5381.4, 6241.4, 6255.4, 6316.2, 6411.6, 6856.1, $7158.8,7274.5,7872.1,9742$ and $12227.3 \mathrm{Da}$ ) were used as external calibrants of the MALDI-TOF MS equipment (Passarini et al., 2013). The mass range from 2000 to $20000 \mathrm{Da}$ was recorded using the linear mode with a delay of $104 \mathrm{~ns}$ and an acceleration voltage of $+20 \mathrm{kV}$. Final spectra were generated by summing 20 laser shots accumulated per profile and 50 profiles produced per sample, leading to 1000 laser shots per summed spectrum. The resulting peak lists were exported to the database of the SARAMIS package software (Spectral Archiving and Microbial Identification System, AnagnosTec, Germany, www.anagnostec.eu) where the final identifications were achieved. Identifications by the SARAMIS package are based on the presence or absence of each peak in the spectra.

\section{MALDI-TOF MS clustering analysis}

Cluster analyses of the MALDI-TOF MS spectral data were achieved using the SARAMIS database software.
In this database peak lists of individual samples were compared to matching SuperSpectra and/or reference spectra generated by the system. For SuperSpectra and reference spectra, SARAMIS uses a point system based on peak list with mass signals weighed according to their specificity. The weighting is based on empirical data from multiple samples of type, reference and well characterised yeast strains. SuperSpectra are consensus spectra containing a pattern of mass signals, which are taxa-specific and allow the identification of strains and cluster analyses of spectra of multiple samples. In present study superspectra for $C$. albicans, C. parapsilosis, C. tropicalis and P. kudriavzevii were used. Reference spectra of C. albicans CBD 924, $C$. parapsilosis CBD 1325 and C. tropicalis CBD 957 were used as individual empiric spectra of well characterised yeast species. In both cases, the similarity between individual spectra is expressed as a relative or an absolute number of matching mass signals after subjecting the data to a single link agglomerative clustering algorithm. The database included SuperSpectra and/or reference spectra of all the yeasts described herein.

\section{ITS sequencing and sequence analysis}

Isolates that presented distinctive identification by the classical and new spectral phenotypic approaches were analysed by ITS sequencing. ITS region was chosen taking into consideration that this region is formally the primary fungal barcode marker recommended by the Consortium for the Barcode of Life (Schoch et al., 2012). Sequence analysis was carried out on the entire ribosomal ITS region (i.e., ITS1/58S rDNA/ITS2), according to White et al. (1990). Sequencing was performed with an ABI 310 Genetic Analyser (Applied Biosystems) using standard protocols. Strains were identified at the level of $99.0 \%$ sequence similarity or higher after a NCBI blast.

\section{Results}

\section{Classical phenotypic identification}

Forty yeast isolates from clinical cases were identified using classic phenotypic identification (Table 1). Identities were as follow: 20 C. albicans, 5 C. krusei, $11 \mathrm{C}$. parapsilosis and 4 C. tropicalis. Sexual reproduction was not observed on Gorodkowa agar although, in contrast, chlamydospores were observed from all C. albicans isolates. In order to clarify identification difficulties on isolates that presented ambiguity, URM 4990, 4388, 4124, 4818,4261 and 1150, VITEK-2 XL was used. This method corroborated all previous phenotypic identifications for these 6 isolates.

\section{MALDI-TOF MS identification}

Six isolates (15\%) identified by MALDI-TOF MS did not correspond to the phenotypic identification (Table 1). The C. krusei isolates were classified as Pichia kudriavzevii 


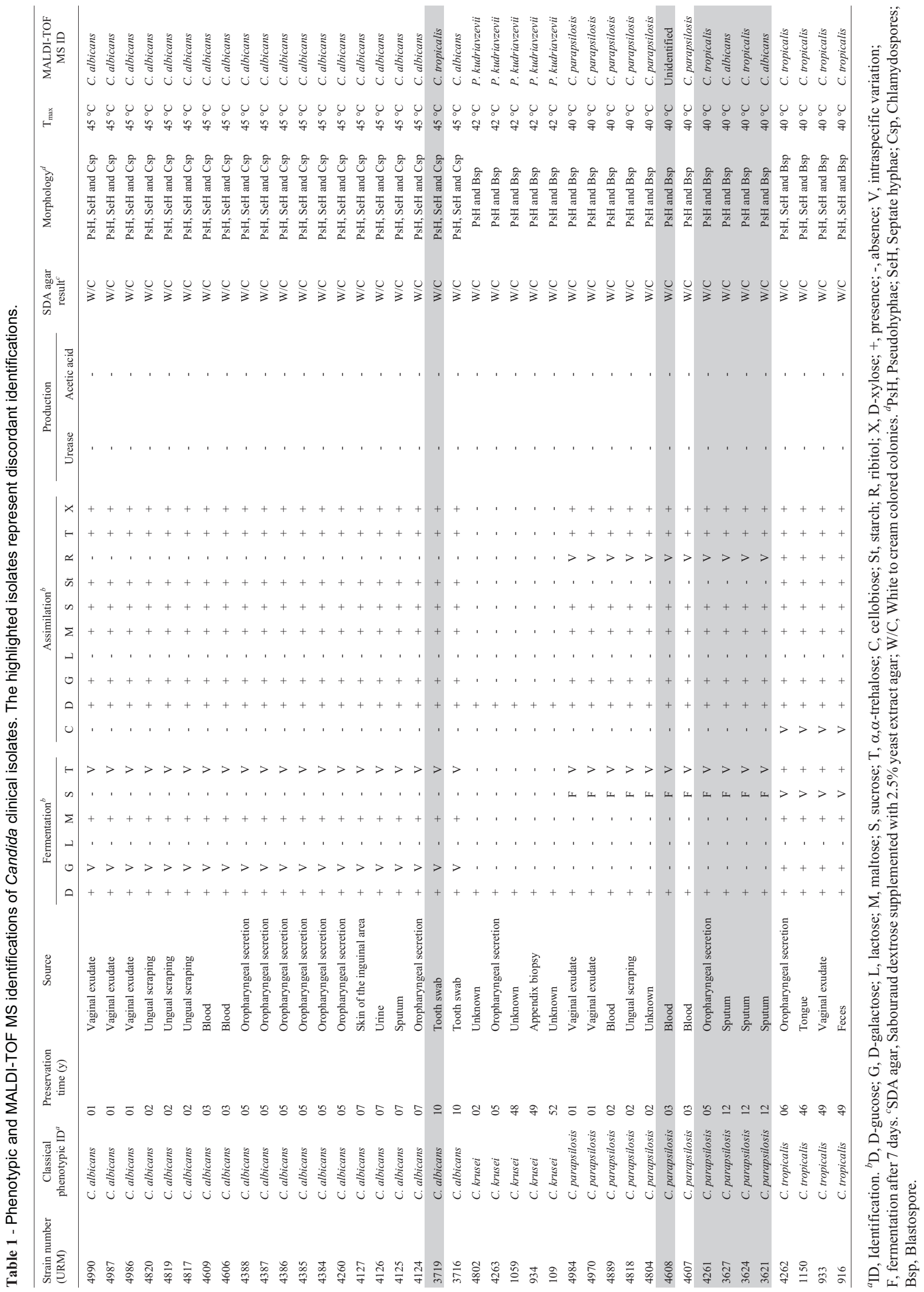




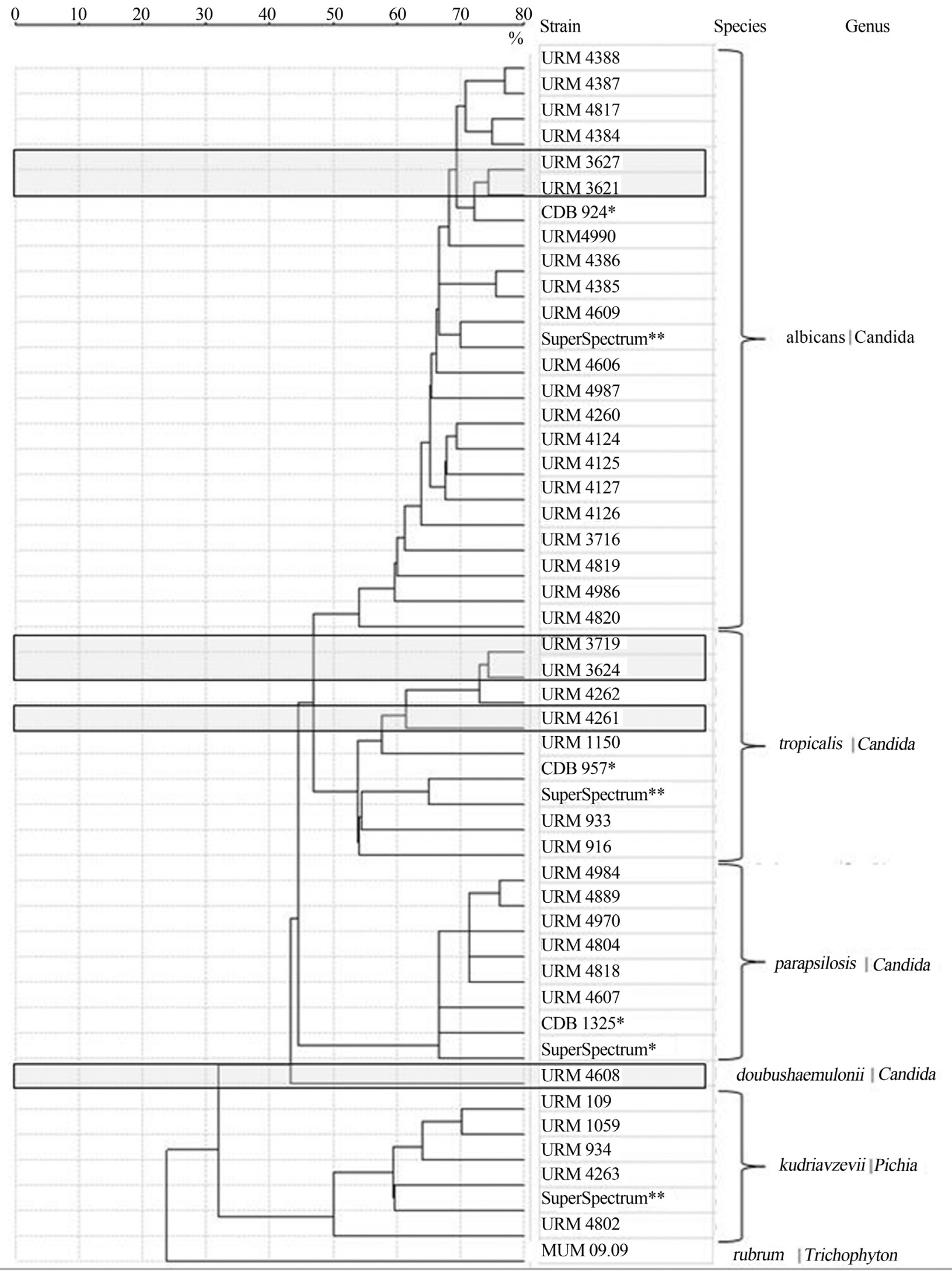

Figure 1 - MALDI-TOF spectra based dendrogram of yeast isolates evaluated in this work. Discordances obtained by MALDI-TOF MS and classical phenotypic analyses are highlighted by grey boxes. CBD reference strains and Superspectra are marked by * and **, respectively. Trichophyton rubrum MUM 09.09 was used as outgroup. 
(former Issatchenkia orientalis) using the SARAMIS software package, which is the basionym teleomorphic name.

Isolates URM 4261, 3627, 3624 and 3621 were identified as $C$. parapsilosis by phenotype. However, URM 4261 and 3624 were C. tropicalis and URM 3627 and 3621 were $C$. albicans by MALDI-TOF MS. Isolate URM 4261 was one of the six samples confirmed by VITEK-2 XL as C. parapsilosis. This finding corresponds to $16.7 \%$ of discordance in the overall isolates analysed by VITEK-2 XL in relation to the spectral phenotypic identification.

URM 4608 was identified as $C$. parapsilosis phenotypically and was recorded as "unidentified" by MALDITOF MS, grouping separately on the dendrogram (Figure 1). This separation was confirmed by the molecular biology results presented below.

Finally, URM 3719 was identified as C. albicans phenotypically and as C. tropicalis by MALDI-TOF MS.

\section{ITS identification}

All six isolates that presented discordant results from phenotypic and MALDI-TOF MS identifications were analysed by ITS sequencing. URM 3621 and 3627 isolates were identified as $C$. parapsilosis by phenotype and $C$. albicans by MALDI-TOF MS (Table 1). ITS sequencing confirmed that these clinical yeast strains were $C$. albicans (URM 3627, accession $n^{\circ}$ KF031307). URM 4261 (accession $n^{\circ}$ KF031306) and 3624 (accession $n^{\circ}$ KF031304) were confirmed as $C$. tropicalis by ITS sequencing.

URM 3719 was identified as C. albicans by phenotype but grouped with $C$. tropicalis by MALDI-TOF MS (Table 1 and Figure 1), and was confirmed as C. tropicalis by ITS analysis (accession $n^{\circ}$ KF031305).

URM 4608 that was identified as $C$. parapsilosis by phenotype could not be identified by MALDI-TOF MS, grouping separately from the $C$. parapsilosis cluster in the MALDI-TOF MS dendrogram (Figure 1). However, it was identified as $C$. doubushaemulonii by ITS (accession $\mathrm{n}^{\circ}$ KF031310) and when this new taxon information was added to the SARAMIS database, the isolate was recorded as C. doubushaemulonii (Figure 1).

Although the good concordance between phenotypical and spectral identification of Pichia kudriavzevii, strains URM 1059 and URM 4802 were chosen for additional ITS analysis. The sequencing results confirmed their identifications (accession n $^{\circ}$ KF031308 and KF031309, respectively).

\section{Discussion}

A comparison between the discriminative capability of phenotypic and MALDI-TOF MS characters of 40 clinical yeasts was reported herein. Moreover, discordant isolates were analysed by ITS sequencing which confirmed the MALDI-TOF MS identifications. Phenotype grouped the isolates into four distinct species: C. albicans, C. krusei (P. kudriavzevii), C. parapsilosis and C. tropicalis. In addi- tion, 6 out of $40(15 \%)$ strains analysed by this method disagreed with the identification performed by MALDI-TOF MS. In line with described by Santos et al. (2011) the MALDI-TOF MS results were confirmed strongly by the ITS sequencing analyses. Through these combined approaches five strains were renamed and one additional species was added to the collection: $C$. doubushaemulonii. The current data, when combined with data previously described (Marinach-Patrice et al., 2010; Jensen and Arendrup, 2011; Santos et al., 2011), indicate that MALDI-TOF MS analysis is a powerful technique to identify clinical Candida isolates.

Because ribosomal proteins can be easily used as biomarkers in the proteomic-based technique by MALDITOF MS the reliability of this methodology is high and can discriminate between closely related Candida species. However, due to the biological variability of these yeasts, even for a single species, the availability of an extensive database is required. For example, MALDI-TOF MS generated a distinctive spectrum for $C$. doubushaemulonii URM 4608 , separating it from the other isolates in the dendrogram, which is otherwise difficult to do. In this case, the database was not sufficiently extensive to provide straightforward the correct identification due to a complete lack of data related with $C$. doubushaemulonii. As a matter of consequence, the culture collections with high quality biological materials are key elements to feed the MALDI-TOF MS databases with reference spectra. In contrast, with this new approach collections gain the possibility to requalify their holdings renaming misidentified strains.

MALDI-TOF MS is straightforward, rapid and employs partially automated procedures. Results are obtained in approximately $30 \mathrm{sec}$ per sample. Since the time for pathogen identification is an important determinant of infection-related mortality rates of hospitalised patients, this technique is an important tool in the fight to reduce mortalities when the time taken for classical methods is considered.

In summary, the classical phenotypic approaches, including VITEK techniques are very important in the daily routine analyses in health centres and hospitals. However, it is associated with a high degree of misidentifications (Lockhart et al., 2008; Marklein et al., 2009; Putignani et al., 2011) and it would be beneficial for these to be supported by reference strains provided by culture collections and, if available, by MALDI-TOF MS with an appropriate database. MALDI-TOF MS is important to improve the efficiency of the classical phenotypical methods for clinical Candida polyphasic identifications even when isolates where preserved for decades in culture collections.

\section{Acknowledgments}

Research leading to these results received partial funding from the European Community's Seventh Framework Program (FP7, 2007-2013), Research Infrastructures 
Action, under grant agreement No. FP7-228310 (EMbaRC project). Thanks are also due to Coordenação de Aperfeiçoamento de Pessoal de Nível Superior (CAPES, Brazil) for funding support.

\section{References}

Barnett JA, Paine RW, Yarrow D (2000) Yeasts: Characteristics and Identification. Cambridge University Press, Cambridge, U.K.

Boundy-Mills K (2012) Yeast culture collections of the world: meeting the needs of industrial researchers. J Ind Microbiol Biotechnol 39:673-680.

Cendejas-Bueno E, Gomez-Lopez A, Mellado E, Rodriguez-Tudela RL, Cuenca-Estrella M (2010) Identification of pathogenic rare yeast species in clinical samples: Comparison between phenotypical and molecular methods. J Clin Microbiol 48:895-1899.

Correia A, Sampaio P, James S, Pais C (2006) Candida bracarensis sp. nov., a novel anamorphic yeast species phenotypically similar to Candida glabrata. Int J Syst Evol Microbiol 56:313-317.

De Hoog GS, Guarro J, Gené J, Figueras MJ (2000) Atlas of clinical fungi. Centraalbureau voor Schimmelculures, Universitat Rovira i Virgili, Utrecht, Reus, The Netherlands.

Essendoubi M, Toubas D, Bouzaggou M, Pinon JM, Manfait M, Sockalingum GD (2005) Rapid identification of Candida species by FT-IR microspectroscopy. Biochim Biophys Acta 1724:239-247.

Holland RD, Wilkes RD, Ralli F, Sutherland RD, Persons CC, Voorhees KJ, Lay JJO (1996) Rapid identification of intact whole bacteria based on spectral patterns using matrixassisted laser desorption/ionization with time-of-flight mass spectrometry. Rapid Commun Mass Spectrom 10:12271232.

Jensen RH, Arendrup MC (2011) Candida palmioleophila: Characterization of a previously overlooked pathogen and its unique susceptibility profile in comparison with five related species. J Clin Microbiol 49:549-556.

Latouche GN, Daniel HM, Lee OC, Mitchell TG, Sorrell TC, Meyer WM (1997) Comparison of use of phenotypic and genotypic characteristics for identification of species of the anamorph genus Candida and related teleomorph yeast species. J Clin Microbiol 35:3171-3180.

Leaw SN, Chang HC, Sun HF, Barton R, Bouchara JP, Chang TC (2006) Identification of medically important yeast species by sequence analysis of the internal transcribed spacer regions. J Clin Microbiol 44:693-699.

Lockhart SR, Messer SA, Pfaller MA, Diekema DJ (2008) Lodderomyces elongisporus masquerading as Candida parapsilosis as a cause of bloodstream infections. J Clin Microbiol 46:374-376.

Macedo DPC, Farias AMA, Lima-Neto RG, Silva VKA, Leal AFG, Neves RP (2009) Opportunistic yeast infections and enzymatic profile of the etiological agents. Rev Soc Bras Med Trop 42:188-191.

Marinach-Patrice C, Fekkar A, Atanasova R, Gomes J, Djamdjian L, Brossas JY, Meyer I, Buffet P, Snounou G, Datry A, Hennequin C.; Golmard JL, Mazier D (2010) Rapid species diagnosis for invasive candidiasis using mass spectrometry. PLOS ONE 5:1-5.
Marklein G, Josten M, Klanke U, Müller E, Horré R, Maier T, Wenzel T Kostrzewa M, Bierbaum G, Hoerauf A, Sahl HG (2009) Matrix-assisted laser desorption ionization-time of flight mass spectrometry for fast and reliable identification of clinical yeast isolates. J Clin Microbiol 47:2912-2917.

Medrano DJA, Brilhante RSN, Cordeiro RA, Rocha MFG, Rabenhorst SHB; Sidrim JJC (2006) Candidemia in a Brazilian hospital: The importance of Candida parapsilosis. Rev Inst Med Trop S Paulo 48:17-20.

Mozina SS, Raspor P (1997) Molecular techniques for food identification in food processing. Food Technol Biotechnol 35:55-61.

OECD (2007) Best Practices Guidelines for Biological Resource Centres. Available at: http://www.oecd.org/sti/biotechnologypolicies/38777417.p df. Accessed 14 December 2012.

Passarini MRZ, Santos C, Lima N, Berlinck RGS, Sette LD (2013) Filamentous fungi from the Atlantic marine sponge Dragmacidon reticulatum. Arch Microbiol 195:99-111.

Putignani L, Del Chierico F, Onori M, Mancinelli L, Argentieri M, Bernaschi P, Coltella L, Lucignano B, Pansani L, Ranno S, Russo C, Urbani A, Federicibd G, Menichella D (2011) MALDI-TOF mass spectrometry proteomic phenotyping of clinically relevant fungi. Mol BioSyst 7:620-629.

Qian J, Cutler JE, Cole RB, Cai Y (2008) MALDI-TOF mass signatures for differentiation of yeast species, strain grouping and monitoring of morphogenesis markers. Anal Bioanal Chem 392:439-449.

Santos C, Fraga ME, Kozakiewicz Z, Lima N (2010b) Fourier transform infrared as a powerful technique for the identification and characterisation of filamentous fungi and yeasts. Res Microbiol 161:168-175.

Santos C, Lima N, Sampaio P, Pais C (2011) Matrix-assisted laser desorption/ionization time-of-flight intact cell mass spectrometry (MALDI-TOF-ICMS) to detect emerging pathogenic Candida species. Diagn Microbial Infect Dis 71:304308.

Santos C, Paterson RMR, Venâncio A, Lima N (2010a) Filamentous fungal characterisations by matrix-assisted laser desorption/ionisation time of flight mass spectrometry. J Appl Microbiol 108:375-385.

Schoch CL, Seifert KA, Huhndorf S, Robert V, Spouge JL, Levesque CA, Chen W, Fungal Barcoding Consortium Author List (2012) Nuclear ribosomal internal transcribed spacer (ITS) region as a universal DNA barcode marker for Fungi. Proc Natl Acad Sci USA, 109:6241-6246.

Simões MF, Pereira L, Santos C, Lima N (2013) Polyphasic identification and preservation of fungal diversity: Concepts and applications. In: Malik, A., Grohmann, E., Alves, M.(eds). Management of Microbial Resources in the Environment. Springer, New York, USA, p. 91-117.

Smith D (2012) Culture collections. In: Sariaslani, S., Gaad, GM.(eds). Advances in Applied Microbiology. Academic Press, Burlington, USA, p. 73-118.

Sullivan DJ, Westerneng TJ, Haynes KA, Bennett DE, Coleman DC (1995) Candida dubliniensis sp. nov.: phenotypic and molecular characterization of a novel species associated with oral candidosis in HIV-infected individuals. Microbiology 141:1507-1521.

Tavanti A, Davidson AD, Gow NAR, Maiden MCJ, Odds FC (2005) Candida orthopsilosis and Candida metapsilosis 
spp. nov. to replace Candida parapsilosis groups II and III. J Clin Microbiol 43:284-292.

van Veen SQ, Claas ECJ, Kuijper EJ (2010) High-throughput identification of bacteria and yeast by matrix-assisted laser desorption ionization-time of flight mass spectrometry in conventional medical microbiology laboratories. J Clin Microbiol 48:900-907.
White TJ, Burns T, Lee S, Taylor J (1990) Amplification and direct sequencing of fungal ribosomal RNA genes for phylogenetics. In: Innis, M.A., Gelfand, D.H., Sninsky, J.J., Taylor, T.J.(eds.). PCR Protocols: a Guide for Methods and Applications. Academic Press, New York, USA, p. 315-322.

All the content of the journal, except where otherwise noted, is licensed under a Creative Commons License CC BY-NC. 